\title{
Strong gravitational lensing: Why no central black holes?
}

\author{
Da-Ming Chen ${ }^{\star}$
}

\author{
National Astronomical Observatories, Chinese Academy of Sciences, Beijing 100012, PR China
}

Received 12 July 2002 / Accepted 26 August 2002

\begin{abstract}
We investigate how central black holes (BHs) in galactic dark halos could affect strong gravitational lensing. The distribution of integral lensing probability with image separations are calculated for quasars of redshift 1.5 by foreground dark matter halos. The mass density of dark halos is taken to be the Navarro-Frenk-White (NFW) profile such that, when the mass of a halo is less than $10^{14} M_{\odot}$, its central black holes or a bulge is included as a point mass. The relationship between the masses $M$. of supermassive black holes and the total gravitational mass $M_{\mathrm{DM}}$ of their host galaxy is adopted from the most recent literature. Only a flat $\Lambda \mathrm{CDM}$ model is considered here. It is shown that, while a single black hole for each galaxy contributes considerable but not sufficient lensing probabilities at small image separations compared with those without black holes, the bulges (which are about 100-1000 times larger in mass than a typical black hole) would definitely contribute enough probability at small image separations, although it gives too high probabilities at large separation angles compared with lensing observations.
\end{abstract}

Key words. gravitational lensing - physics black holes - galaxies: bulges

\section{Introduction}

Cold Dark Matter (CDM) has become the standard theory of cosmological structure formation. The $\Lambda \mathrm{CDM}$ variant of CDM with $\Omega_{\mathrm{m}}=1-\Omega_{\Lambda} \approx 0.3$ appears to be in good agreement with the available data on large scales (Primack 2002). On smaller (sub-galactic) scales, there seem to be various discrepancies, such: N-body CDM simulations which give cuspy halos with divergent profiles towards the center (Navarro et al. 1996, 1997, NFW hereafter); bar stability in high surface brightness spiral galaxies which also demands low-density cores; CDM models which yield an excess of small scale structures; formation of disk galaxy angular momentum, which is much too small in galaxy simulations. Issues that have arisen on smaller scales have prompted people to propose a wide variety of alternatives to CDM, such as warm dark matter (WDM) and selfinteracting dark matter (SIDM). Now that problems arise from galaxy-size halos and centers of all dark matter halos, highresolution simulations and observations are the final criterion. Recent highest-resolution simulations appear to be consistent with NFW (Klypin 2002; Power et al. 2002) until scales smaller than about $1 \mathrm{kpc}$. Meanwhile, a large set of high-resolution optical rotation curves has recently been analyzed for low surface brightness (LSB) galaxies. One can also conclude that the NFW profile is a good fit down to about $1 \mathrm{kpc}$. Although further simulations and observations, including measurement of $\mathrm{CO}$ rotation curves (Bolatto et al. 2002), may help to clarify the nature of the dark matter, it now appears that WDM and

\footnotetext{
* e-mail: cdm@class1.bao.ac.cn
}

SIDM are both probably ruled out, while the small-scale predictions of $\Lambda \mathrm{CDM}$ may be in better agreement with the latest data than appeared to be the case as recently as a year ago.

In addition to direct simulations and observations, gravitational lensing provides another powerful probe of mass distribution in the universe. Since mass within small scales only deflect light rays slightly, it is difficult to extract mass information from a single lensing event, and thus statistical gravitational lensing is needed even for "strong" gravitational lensing of small halos (Turner et al. 1984; Narayan \& White 1988; Cen et al. 1994; Kochanek 1995; Wambsganss et al. 1995; Wambsganss et al. 1998; Porciani \& Madau 2000; Keeton \& Madau 2001). Li \& Ostriker (2002) first used the semianalytical approach to analyze gravitational lensing of remote quasars by foreground dark halos in various cold dark matter cosmologies. The mass function of dark halos they used is alternatively given by singular isothermal sphere (SIS), the NFW profile, or the generalized NFW profile. They found that none of these models can completely explain the current observations: the SIS models predict too many large splitting lenses, while the NFW models predict too few small splitting lenses, so they proposed that there must be at least two populations of halos in the universe: small mass halos with a steep inner density slope and large mass halos with a shallow inner density slope. The author conclude that a combination of SIS and NFW halos can reasonably reproduce the current observations. Similarly, Sarbu et al. (2001) investigated the statistics of gravitational lenses in flat, low-density cosmological models with different cosmic equations of state $\omega$. It was found 
that COBE-normalized models with $\omega>-0.4$ produce too few arcsecond-scale lenses in comparison with the JVAS/CLASS radio survey, a result that is consistent with other observational constraints on $\omega$.

When attention is attracted to alternatives of CDM dark matter density profile at small scales, another kind of dark matter - super-massive black holes in the centers of most galactic halos is forgotten or ignored in this case, although the idea of detecting supermassive compact objects by their gravitational lensing effects was proposed very early (Press \& Gunn 1973; Wilkinson et al. 2001) and the lensing effects of Schwarzschild black holes in the strong field regime have been discussed in detail (e.g., Virbhadra \& Ellis 2000; Frittelli et al. 2000; Bozza et al. 2001). On the other hand, cosmological voids can form directly after the collapse of extremely large wavelength perturbations into low-density black holes or cosmological black holes; such black holes can also be detected through their weak and strong lensing effects (Stornaiolo 2002). The observational evidence presented so far suggests the ubiquity of BHs in the nuclei of all bright galaxies, regardless of their activity, and BH masses correlate with masses and luminosities of the host spheroids and, more tightly, with stellar velocity dispersions (Magorrian et al. 1998; Ferrarese \& Merritt 2000; Ravindranath et al. 2001; Merritt \& Ferrarese 2001a, 2001b; Wandel 2002; Sarzi et al. 2002). Most recent high-resolution observational data gives $M_{\bullet} / M_{\text {bulge }} \approx 10^{-3}$ (Merritt \& Ferrarese 2001c). Ferrarese (2002) further gave the relation between masses $M$. of supermassive black holes and the total gravitational mass of the dark matter halo in which they presumably formed

$$
\frac{M_{\bullet}}{10^{8} M_{\odot}} \sim 0.046\left(\frac{M_{\mathrm{DM}}}{10^{12} M_{\odot}}\right)^{1.57} \text {. }
$$

In this paper, we investigate the contributions of galactic central black holes to lensing probabilities at small image separations. Since $\Lambda$ CDM cosmology and NFW profile are in good agreement with the available data of structure formation on almost all scales as mentioned above, we only chose these two models respectively as cosmology and mass density function in our calculations. We model the lenses as a population of dark matter halos with an improved version of the Press-Schechter (1974, PS) mass distribution function, and central BHs are considered for galaxy-size halos.

The paper is organized as follows: the lensing equation is given in Sect. 2, lensing probabilities are calculated in Sect. 3, and discussion and conclusions are provided in Sect. 4.

\section{Lensing equation}

The NFW profile is

$\rho_{\mathrm{NFW}}=\frac{\rho_{\mathrm{s}} r_{\mathrm{s}}^{3}}{r\left(r+r_{\mathrm{s}}\right)^{2}}$

where $\rho_{\mathrm{s}}$ and $r_{\mathrm{s}}$ are constants. We can define the mass of a halo to be the mass within $r_{200}$ (which is the radius of a sphere around a dark halo within which the average mass density is 200 times the critical mean mass density of the universe),

$M_{\mathrm{DM}}=4 \pi \int_{0}^{r_{200}} \rho r^{2} \mathrm{~d} r=4 \pi \rho_{\mathrm{s}} r_{\mathrm{s}}^{3} f\left(c_{1}\right)$, with $c_{1}=r_{200} / r_{\mathrm{s}}$ the concentration parameter, the value of which is chosen to be $7 /(1+z)$ (Bartelmann el al. 1998). And

$f\left(c_{1}\right)=\int_{0}^{c_{1}} \frac{x^{2} \mathrm{~d} x}{x(1+x)^{2}}=\ln \left(1+c_{1}\right)-\frac{c_{1}}{1+c_{1}}$.

In flat $\Lambda \mathrm{CDM}$ cosmology, the constants $\rho_{\mathrm{s}}$ and $r_{\mathrm{s}}$ can then be expressed as (Li \& Ostriker 2002),

$\rho_{\mathrm{s}}=\rho_{\text {crit }}\left[\Omega_{\mathrm{m}}(1+z)^{3}+\Omega_{\Lambda}\right] \frac{200}{3} \frac{c_{1}^{3}}{f\left(c_{1}\right)}$,

$r_{\mathrm{s}}=\frac{1.626}{c_{1}} \frac{M_{15}^{1 / 3}}{\left[\Omega_{\mathrm{m}}(1+z)^{3}+\Omega_{\Lambda}\right]^{1 / 3}} h^{-1} \mathrm{Mpc}$

where $\rho_{\text {crit }}$ is the present value of the critical mass density of the universe, and $M_{15}$ is the reduced mass of a halo defined as $M_{15}=M_{\mathrm{DM}} /\left(10^{15} h^{-1} M_{\odot}\right)$.

The surface mass density for NFW profile is

$\Sigma_{\mathrm{NFW}}(x)=2 \rho_{\mathrm{s}} r_{\mathrm{s}} \times \begin{cases}\frac{\ln x-\sqrt{1-x^{2}}-\ln \left(1-\sqrt{1-x^{2}}\right)}{\left(1-x^{2}\right)^{3 / 2}}, & (x>1), \\ \frac{1}{3}, & (x=1), \\ \frac{\arcsin (1 / x)+\sqrt{x^{2}-1}-\pi / 2}{\left(x^{2}-1\right)^{3 / 2}}, & (0<x<1) .\end{cases}$

Where $x=|\boldsymbol{x}|$ and $\boldsymbol{x}=\boldsymbol{\xi} / r_{\mathrm{s}}, \boldsymbol{\xi}$ is the position vector in the lens plane. The galactic central black holes are assumed to be point masses, and we consider fist there is only a single black hole with mass $M_{\bullet}$ for each galaxy. So the surface mass density for galactic halos each with a single central black hole can be written as

$\Sigma_{\text {galaxy }}(\boldsymbol{x})=M_{\bullet} \delta^{2}(\boldsymbol{x})+\Sigma_{\mathrm{NFW}}(\boldsymbol{x})$,

where $\delta^{2}(\boldsymbol{x})$ is the two dimensional Dirac-delta function. The lensing equation with galactic central black holes considered then is

$y=x-\mu_{\mathrm{s}} \frac{f_{\mathrm{BH}}+g(x)}{x}$,

where $y=|\boldsymbol{y}|, \boldsymbol{\eta}=\boldsymbol{y} D_{\mathrm{S}}^{\mathrm{A}} / D_{\mathrm{L}}^{\mathrm{A}}$ is the position vector in the source plance, in which $D_{\mathrm{S}}^{\mathrm{A}}$ and $D_{\mathrm{L}}^{\mathrm{A}}$ are angular-diameter distances from the observer to the source and to the lens respectively. And

$\mu_{\mathrm{s}}=\frac{4 \rho_{\mathrm{s}} r_{\mathrm{s}}}{\Sigma_{\mathrm{cr}}}$

where $\Sigma_{\mathrm{cr}}=\left(c^{2} / 4 \pi G\right)\left(D_{\mathrm{S}}^{\mathrm{A}} / D_{\mathrm{L}}^{\mathrm{A}} D_{\mathrm{LS}}^{\mathrm{A}}\right)$ is the so called critical surface mass density, in which $D_{\mathrm{LS}}^{\mathrm{A}}$ is the angular-diameter distance from the lens to the source. And

$g(x)=\ln \frac{x}{2}+ \begin{cases}\frac{\arctan \sqrt{x^{2}-1}}{\sqrt{x^{2}-1}} & (x>1), \\ 1 & (x=1), \\ \frac{\operatorname{arctanh} \sqrt{1-x^{2}}}{\sqrt{1-x^{2}}} & (0<x<1) .\end{cases}$

In Eq. (9), the term $f_{\mathrm{BH}}$ stands for the contribution of a black hole, and by using Eqs. (1) and (3), it has the form

$f_{\mathrm{BH}}=2.78 \times 10^{-4} f\left(c_{1}\right) M_{15}^{0.57}$.

Since there is always more than one black holes in a bulge, and thus the bulge itself can act like a black hole, we can treat a 


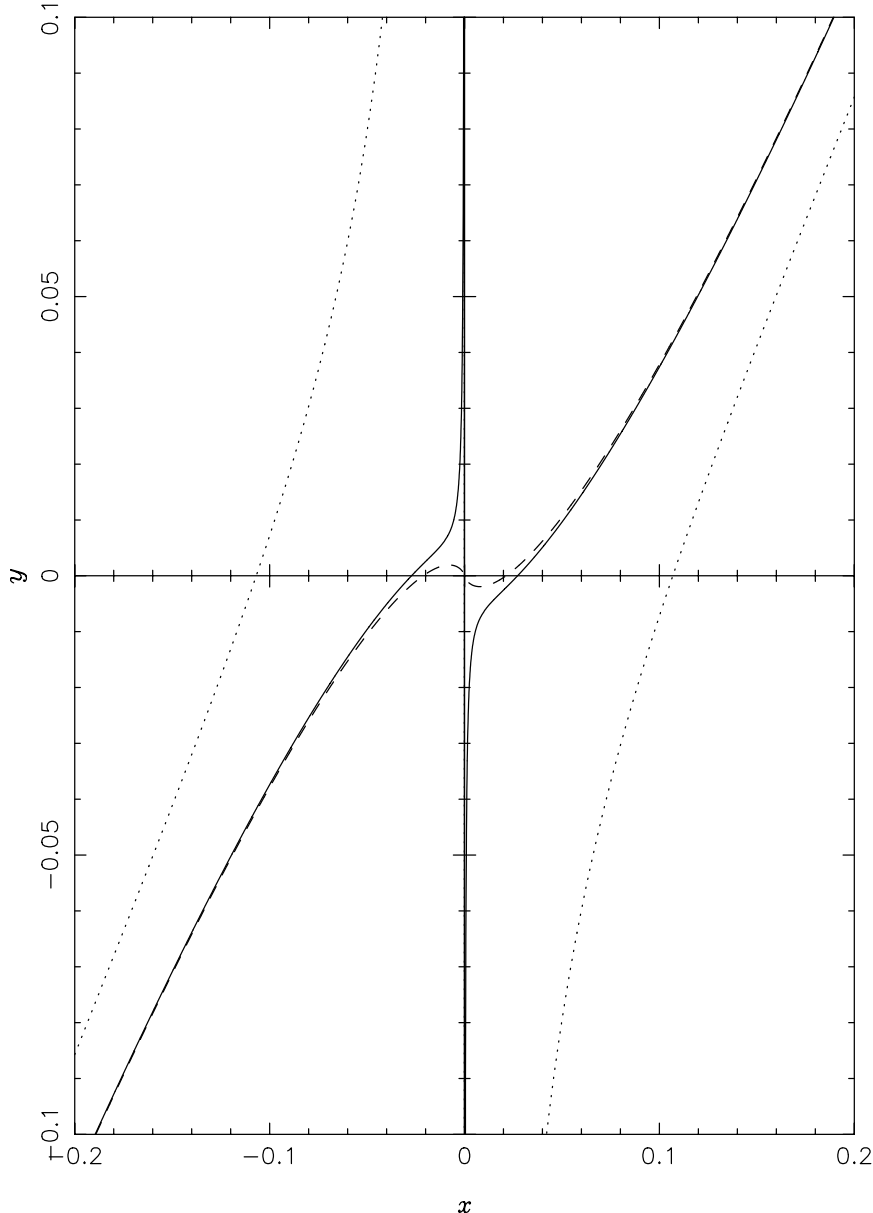

Fig. 1. Lensing equations: dashed line stands for a NFW lens with no central black holes; solid line stands for a lens with a NFW density profile plus a single central black hole; dotted line stands for a lens of NFW plus a bulge (treated as a point mass, an effective black hole).

bulge as a point mass in this paper as an extreme case. The mass of a black hole correlates linearly with that of its host bulge as $M_{\bullet} / M_{\text {bulge }} \approx 10^{-3}$, so we can simply multiply the term $f_{\mathrm{BH}}$ by $10^{3}$ to stand for the contribution of a bulge. However, some light rays from the source will definitely travel across the bulge, so there must exist a kind of "effective" black hole with mass larger than a single "real" black hole but less than the bulge. In order to investigate the tendency of image separations contributed by different point mass, we can multiply $f_{\mathrm{BH}}$ by, for example, $10^{2}$, etc.

The lensing equations for three cases are plotted in Fig. 1 according to Eq. (9), where we have extended $x$ and $y$ to their opposite values because of symmetry. The full line, dashed line and dotted line, respectively, represent the NFW lens with $f_{\mathrm{BH}}=0$, "NFW+BH" lens with $f_{\mathrm{BH}}=2.78 \times 10^{-4} f\left(c_{1}\right) M_{15}^{0.57}$ and "NFW+bulge" with $f_{\mathrm{BH}}=2.78 \times 10^{-2} f\left(c_{1}\right) M_{15}^{0.57}$, in which $\mu_{\mathrm{s}}=0.49$ and $f\left(c_{1}\right)=0.91$.

We find that, as point masses, both a single central black hole and a bulge can more often produce small separation images than the case when no central black holes are considered. This result will be further confirmed by the lensing probability given in next section.

\section{Lensing probability}

We choose the most generally accepted values of the parameters for flat $\Lambda \mathrm{CDM}$ cosmology, for which, with usual symbols, the matter density parameter, vacuum energy density parameter and Hubble constant are respectively: $\Omega_{\mathrm{m}}=0.3, \Omega_{\Lambda}=0.7$, $h=0.75$.

The quasars of redshift $z_{\mathrm{s}}=1.5$ are lensed by foreground CDM halos of galaxy clusters and galaxies, the lensing probability with image separations larger than $\Delta \theta$ is (Schneider et al. 1992)

$$
P(>\Delta \theta)=\int_{0}^{z_{\mathrm{s}}} \frac{\mathrm{d} D_{\mathrm{L}}(z)}{\mathrm{d} z} \mathrm{~d} z \int_{0}^{\infty} \bar{n}(M, z) \sigma(M, z) \mathrm{d} M .
$$

Where $D_{\mathrm{L}}(z)$ is proper distance from the observer to the lens located at redshift $z$

$D_{\mathrm{L}}(z)=\frac{c}{H_{0}} \int_{0}^{z} \frac{\mathrm{d} z}{(1+z) \sqrt{\Omega_{\mathrm{m}}(1+z)^{3}+\Omega_{\Lambda}}}$,

here $c$ is the speed of light in vacuum and $H_{0}$ is the current Hubble constant. The physical number density $\bar{n}(M, z)$ of virialized dark halos of masses between $M$ and $M+d M$ is related to the comoving number density $n(M, z)$ by $\bar{n}(M, z)=$ $n(M, z)(1+z)^{3}$; the latter was originally given by Press \& Schechter (1974), and the improved version is

$n(M, z) \mathrm{d} M=\frac{\rho_{0}}{M} f(M, z) \mathrm{d} M$,

where $\rho_{0}$ is the current mean mass density of the universe, and

$f(M, z)=-\sqrt{\frac{2}{\pi}} \frac{\delta_{c}(z)}{M \Delta} \frac{\mathrm{d} \ln \Delta}{\mathrm{d} \ln M} \exp \left[-\frac{\delta_{c}^{2}(z)}{2 \Delta^{2}}\right]$

is PS mass function. In Eq. (16) above, $\Delta^{2}(M)$ is the present variance of the fluctuations in a sphere containing a mean mass $M$,

$\Delta^{2}(M)=\frac{1}{2 \pi^{2}} \int_{0}^{\infty} P(k) W^{2}\left(k r_{M}\right) k^{2} \mathrm{~d} k$

where $P(k)$ is the power spectrum of density fluctuations, $W\left(k r_{\mathrm{M}}\right)$ is the Fourier transformation of a top-hat window function

$W\left(k r_{\mathrm{M}}\right)=3\left[\frac{\sin \left(k r_{\mathrm{M}}\right)}{\left(k r_{\mathrm{M}}\right)^{3}}-\frac{\cos \left(k r_{\mathrm{M}}\right)}{\left(k r_{\mathrm{M}}\right)^{2}}\right]$,

and

$r_{\mathrm{M}}=\left(\frac{3 M}{4 \pi \rho_{0}}\right)^{1 / 3}$.

In Eq. (16), $\delta_{\mathrm{c}}(z)$ is the over density threshold for spherical collapse by redshift $z$ (Navarro et al. 1997):

$\delta_{\mathrm{c}}(z)=\frac{1.68}{D(z)}$

where $D(z)$ is the linear growth function of density perturbation (Carroll \& Press 1992)

$D(z)=\frac{g(\Omega(z))}{g\left(\Omega_{\mathrm{m}}\right)(1+z)}$, 
in which

$g(x)=\frac{5}{2} x\left(\frac{1}{70}+\frac{209 x}{140}-\frac{x^{2}}{140}+x^{4 / 7}\right)^{-1}$,

and

$\Omega(z)=\frac{\Omega_{\mathrm{m}}(1+z)^{3}}{1-\Omega_{\mathrm{m}}+\Omega_{\mathrm{m}}(1+z)^{3}}$.

We use the fitting formulae for CDM power spectrum $P(k)$ given by Eisenstein \& $\mathrm{Hu}$ (1999)

$P(k)=A k T^{2}(k)$

where $A$ is the amplitude normalized to $\sigma_{8}=\Delta\left(r_{\mathrm{M}}=\right.$ $\left.8 h^{-1} \mathrm{Mpc}\right)=0.95$, and

$T=\frac{L}{L+C q_{\mathrm{eff}}^{2}}$,

with

$L \equiv \ln \left(e+1.84 q_{\mathrm{eff}}\right)$,

$q_{\mathrm{eff}} \equiv \frac{k}{\Omega_{\mathrm{m}} h^{2} \mathrm{Mpc}^{-1}}$,

$C \equiv 14.4+\frac{325}{1+60.5 q_{\mathrm{eff}}^{1.11}}$

We need to know the cross-sections in Eq. (13). Since we are interested in the lensing probabilities with image separations larger than a certain value $\Delta \theta$ (ranging from $0 \sim 10 \operatorname{arcsec}$, for example), the cross-section is defined under the condition that the multiple images can be created. For the lenses with NFW profile, one can see from Fig. 1 that multiple images can be produced only if $|y| \leq y_{\mathrm{cr}}$, where $y_{\mathrm{cr}}$ is the maximum value of $y$ when $x<0$, which is determined by $\mathrm{d} y / \mathrm{d} x=0$ when $f_{\mathrm{BH}}=0$ in Eq. (9). For galaxy-size halos, the mass of which is confined to be less than $10^{14} M_{\odot}$ through out this paper, a central black hole or a bulge as a point mass is considered (see Eq. (9)). In this case, multiple images will always exist: when the source is close to the point caustic, i.e., when $y$ is small, there are three images, two of which are within the Einstein circle and the third one the outside which Einstein circle; when $y$ is large enough, there are two images, the weaker one is close to the center of the Einstein circle and the brighter one locates outside of the Einstein circle. So another condition is needed to define the cross-section, for which we use the brightness ratio between the brighter and weaker images just mentioned, and it is enough to set the ratio to be 10 .

The brightness ratio $r$ for the two images is just the ratio of the corresponding absolute values of magnifications (Schneider et al. 1992),

$r=\left|\frac{\mu_{+}}{\mu_{-}}\right|$,

where

$\mu_{+}(y(x))=\left(\frac{y}{x} \frac{\mathrm{d} y}{\mathrm{~d} x}\right)_{x>0}$, $\mu_{-}(y(x))=\left(\frac{y}{x} \frac{\mathrm{d} y}{\mathrm{~d} x}\right)_{x<0}$.

Once the source position $y_{\mathrm{cr}}$ is determined by

$\left|\mu_{+}\left(y_{\mathrm{cr}}\right)\right|=10\left|\mu_{-}\left(y_{\mathrm{cr}}\right)\right|$,

the cross-section can be calculated, both with and without central black holes, as

$\sigma(M, z)=\pi y_{\mathrm{cr}}^{2} r_{\mathrm{s}}^{2} \vartheta\left(M-M_{\min }\right)$,

where $\vartheta(x)$ is a step function, and $M_{\min }$ is determined by lower limit of image separation

$\Delta \theta=\frac{r_{\mathrm{s}} \Delta x}{D_{\mathrm{L}}^{\mathrm{A}}} \approx \frac{2 x_{0} r_{\mathrm{s}}}{D_{\mathrm{L}}^{\mathrm{A}}}$

and Eq. (6) as

$M_{\min }=8.927 \times 10^{-8} M_{15}\left(\Omega_{\mathrm{m}}(1+z)^{3}+\Omega_{\Lambda}\right)\left(\frac{c_{1} D_{\mathrm{L}}^{\mathrm{A}} \Delta \theta}{x_{0}}\right)^{3}$.

In Eq. (34), we have approximated the image separation $\Delta x$ to be $2 x_{0}$, where $x_{0}$ is the positive zero position of function $y(x)$, both when $f_{\mathrm{BH}}=0$ (for NFW lens only) and $f_{\mathrm{BH}} \neq 0$ (for galactic, NFW+BH/bulge lenses) in Eq. (9), since image separation is insensitive to the source position $y$ (Li \& Ostriker 2002). We plot the lensing probability with image separations larger than $\Delta \theta$ in Fig. 2. In order to show the tendency of contributions to the lensing probability for different fraction of the bulge mass (the so called "effective" black hole), we take the term $f_{\mathrm{BH}}$ in Eq. (9) to be $f_{\mathrm{BH}}=2.78 \times 10^{-1} f\left(c_{1}\right) M_{15}^{0.57}, f_{\mathrm{BH}}=$ $2.78 \times\left(5.0 \times 10^{-2}\right) f\left(c_{1}\right) M_{15}^{0.57}$ and $f_{\mathrm{BH}}=2.78 \times 10^{-2} f\left(c_{1}\right) M_{15}^{0.57}$; they are represented by the first three lines from top down, respectively. When central black holes or bulges are included and treated as point masses, the mass of their host halos is confined to be less than $10^{14} M_{\odot}$, because Eq. (1) strictly applies in the range $10^{6}<M_{\bullet}<2 \times 10^{9} M_{\odot}$ and $10^{14} M_{\odot}$ is the upper-limit of galaxy mass (Ferrarese 2002).

\section{Discussion and conclusions}

Our numerical results for lensing probability with image separations larger than $\Delta \theta$ in five different cases are shown in Fig. 2. In all cases, lensing probabilities keep nearly constant until $\Delta \theta \sim 0.1$ arcsec, and obvious dropdown takes place at about 1 arcsec if central black holes are included, which, of course, does not mean that the main lensing events have image separations larger than 0.1 arcsec. As a matter of fact, in the NFW case (without galactic central black holes, the full line in Fig. 2), the lensing probability drops quite slowly in the whole range of image separations: $\Delta \theta \sim 0-10$ arcsec; such a tendency would extend even to 30 arcsec if it is plotted beyond this range, which implies a uniform distribution of lensing probability for its log value among image separations. However, note that in the single black hole case (dotted line), the lensing probability drops to the same value of NFW at 2 arcsec, which gives the influence range of a single black hole. In the range of $0 \sim 0.1$ arcsec, the lensing probability for the single black hole case is about 3 times that for NFW. 


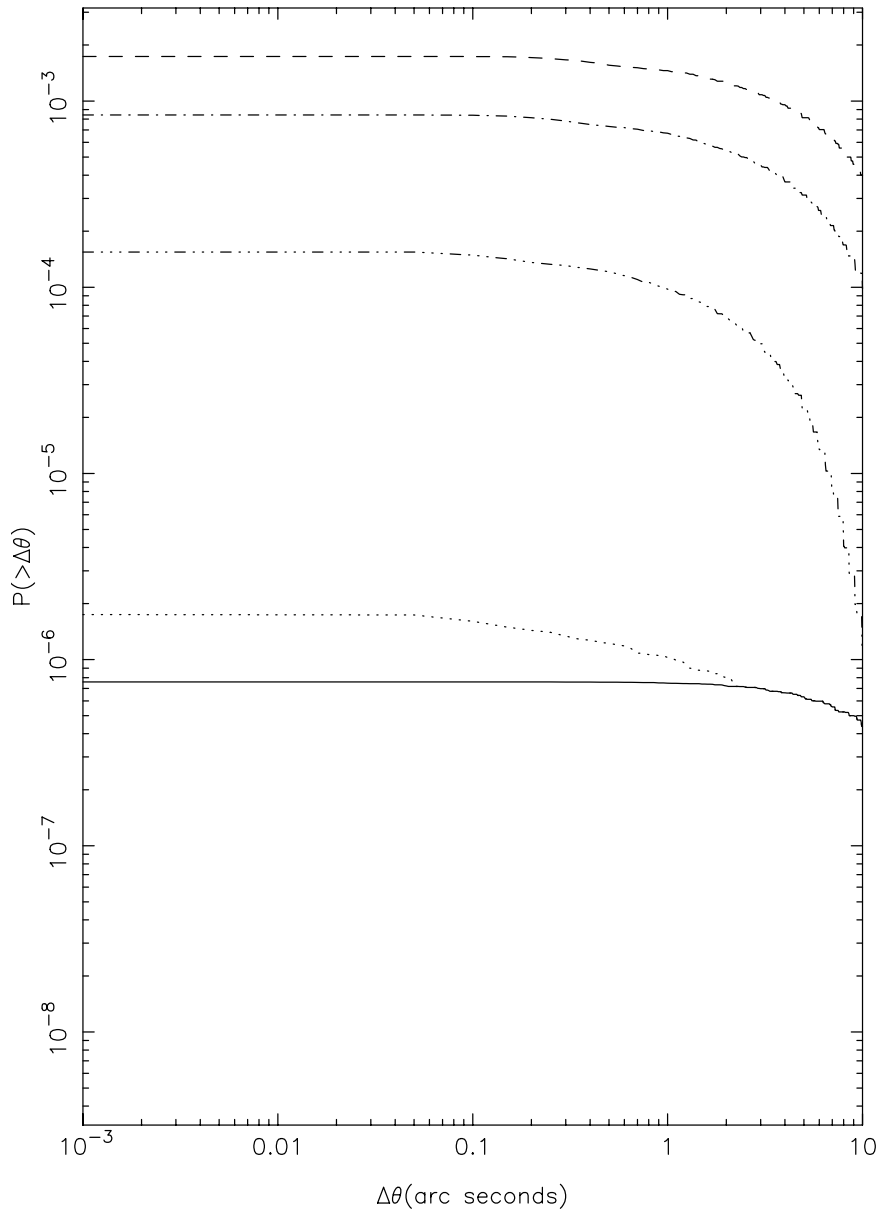

Fig. 2. Lensing probability with image separations larger than $\Delta \theta$ : the full line is for lenses of NFW density profile with no central black holes for halos at all scales, dotted line shows the case when a single central black hole for each galaxy-size halo is included. Other three lines show the cases when collectors of central black holes in the bulge are treated as an effective black hole. The dashed, dot-dash and dashdot-dot-dot line, from top downwards, show respectively, the mass of the effective black hole at 1000, 500 and 100 times that of a single "real" black hole.

So, clearly, the contributions from central black holes cannot be omitted, although such contributions alone are indeed not enough to explain the observational data. As we have pointed out, there is always more than one black hole in a galactic bulge, and the collector of black holes would make a bulge itself "act like" a black hole. On the other hand, not all the mass of a bulge is concentrated in black holes, so if we treat a whole bulge as an extreme black hole, such a model would produce too many lenses at image separations larger than 3 arcsec compared with the JVAS/CLASS radio survey. As mentioned above, we have sufficient reason to tune the fraction of a bulge mass to produce a "right" profile of lensing probability at larger image separations required by observational data, but this "sufficient reason" seems not make us produce sufficient lensing probabilities at smaller image separations, as shown by the dash-dot line in Fig. 2.

However, we can attribute sufficient lens events at small image separations to galactic central black holes or the bulge. On the one hand, since this paper focuses on whether galactic central black holes would contribute considerably to the lensing probability, we have not considered the effect of magnification bias, which would increase the final result provided here at all image separations. On the other hand, we have used an improved version of the PS halo mass function but not the "best" version. The shape of the mass function predicted by standard PS theory (the improved version) is in reasonable agreement with what is measured in numerical simulations of hierarchical clustering from Gaussian initial conditions only for massive halos; less massive halos are more strongly clustered or less anti-biased than the standard PS predicted. Sheth \& Tormen (1999, ST) proposed a model that provides a reasonably good fit to the bias relation of less massive haloes as well as to that of massive halos. Note that central black holes are only found in galactic bulges; ST's correction for mass function in the range of less massive halos would definitely change the lensing probability discussed in this paper. Also note that one of the two images produced by a galactic central black hole is close to the lens center and very faint; however, VLBI experiments can detect its existence (Hirabayashi 1990; Ulvestad 1999), and further radio lensing surveys would have the ability to identify high flux density ratio of the two images. How and to what extent lensing magnification bias, flux ratio and modified PS mass function may change the final result will be discussed in another paper.

Acknowledgements. I thank Professor Xiang-Ping Wu for his original idea for this paper and the anonymous referee for useful comments and suggestions. This work was supported by the National Natural Science Foundation of China.

\section{References}

Bartelmann, M., Huss, A., Colberg, J. M., et al. 1998, A\&A, 330, 1 Bolatto, A. D., Simon, J. D., Leroy, A., \& Blitz, L. 2002, ApJ, 565, 238

Bozza, V., Capozziello, S., Iovane, G., \& Scarpetta, G. 2001, GRG, 33,1535

Carroll, S. M., \& Press, W. H. 1992, ARA\&A, 30, 499

Cen, R., Gott, J. R., Ostriker, J. P., \& Turner, E. L. 1994, ApJ, 423, 1

Eisenstein, D. J., \& Hu, W. 1999, ApJ, 511, 5

Ferrarese, L. 2002, ApJ, in press [astro-ph/0203469]

Ferrarese, L., \& Merritt, D. 2000, ApJ, 539, L9

Frittelli, S., Kling, T. P., \& Newman, E. T. 2000, Phys. Rev. D, 61, 064021

Hirabayashi, H. 1990, VSOP, A space VLBI programme. In Observatories in Earth Orbit and Beyond, ed. Y. Kondo (Dordrecht: Kluwer Academic Publishers), 263

Keeton, C. R., \& Madau, P. 2001, ApJ, 549, L25

Klypin, A., Zhao, H., \& Somerville, R. S. 2002, ApJ, 573, 597

Kochanek, C. S. 1995, ApJ, 453, 545

Li, L. -X., \& Ostriker, J. P. 2002, ApJ, 566, 652

Magorrian, J., Tremaine, S., Richstone, D., et al. 1998, AJ, 115, 2285

Merritt, D., \& Ferrarese, L. 2001a, ApJ, 547, 140

Merritt, D., \& Ferrarese, L. 2001b, MNRAS, 320, L30

Merritt, D., \& Ferrarese, L. 2001c, to appear in the Central Kpc of Starbursts and AGN, ed. J. H. Knapen, J. E. Beckman, I. Shlosman, \& T. J. Mahoney [astro-ph/0107134]

Narayan, R., \& White, S. D. M. 1988, MNRAS, 231, 97 
Navarro, J. F., Frenk, C. S., \& White, S. D. M. 1996, ApJ, 462, 563

Navarro, J. F., Frenk, C. S., \& White, S. D. M. 1997, ApJ, 490, 493

Porciani, C., \& Madau, P. 2000, ApJ, 532, 679

Power, C., Navarro, J. F., Jenkins, A., et al. 2002, MNRAS, submitted [astro-ph/0201544]

Press, W. H., \& Gunn, J. E. 1973, ApJ, 185, 397

Press, W. H., \& Schechter, P. 1974, ApJ, 187, 425

Primack, J. R. 2002 [astro-ph/0205391]

Ravindranath, S., Ho, L., \& Filippenko, A. V. 2002, ApJ, 566, 801

Sarbu, N., Rusin, D., \& Ma, C.-P. 2001, ApJ, 561, L147

Sarzi, M., Rix, H.-W., Shields, J. C., et al. 2002, ApJ, 567, 237

Sheth, R. K., \& Tormen, G. 1999, MNRAS, 308, 119

Schneider, P., Ehlers, J., \& Falco, E. E. 1992, Gravitational Lenses (Berlin: Springer-Verlag)
Stornaiolo, C. 2002, GRG, 34, 2089

Turner, E. L., Ostriker, J. P., \& Gott, J. R. 1984, ApJ, 284, 1

Ulvestad, J. S. 1999, Goals of the ARISE space VLBI Mission, New Astronomy Reviews, Proceedings of the 4th EVN/JIVE Symposium

Virbhadra, K. S., \& Ellis, G. F. R. 2000, Phys. Rev. D, 62, 084003

Wambsganss, J., Cen, R., Ostriker, J. P., \& Turner, E. L. 1995, Science, 268,274

Wambsganss, J., Cen, R., \& Ostriker, J. P. 1998, ApJ, 494, 29

Wandel, A. 2002, ApJ, 565, 762

Wilkinson, P. N., Henstock, D. R., Browne, I. W. A., et al. 2001, Phys. Rev. Lett., 86, 584 